\title{
Heart Morphology Differences Induced by Intrauterine Growth Restriction and Premature Birth Measured on the ECG in Pre-adolescents
}

\author{
Nuria Ortigosa ${ }^{1}$, Fátima Crispi ${ }^{2}$, Raquel Bailón ${ }^{3,4}$, Merida Rodriguez-Lopez ${ }^{2}$, Eduard Gratacós ${ }^{2}$, \\ Sebastián Savari ${ }^{5}$, Marta Sitges ${ }^{5}$, Bart Bijnens ${ }^{6}$, Pablo Laguna ${ }^{3,4}$ \\ ${ }^{1}$ I.U. Matemática Pura y Aplicada, Universitat Politècnica de València, Spain \\ ${ }^{2}$ Fetal i+D Medicine Research Center, BCNatal-Barcelona Center for Maternal-Fetal and Neonatal \\ Medicine (Hospital Clínic and Sant Joan de Deu). IDIBAPS, University of Barcelona, (CIBER-ER). \\ ${ }^{3}$ Aragón Institute of Engineering Research (I3A), IIS Aragón, University of Zaragoza, Spain \\ ${ }^{4}$ CIBER-BBN, Spain \\ ${ }^{5}$ Dept. of Cardiology (Institut Clínic del Tòrax), Hospital Clínic - Institut d'Inv. Biomèdiques August \\ Pi i Sunyer, Univ. Barcelona,Spain. \\ ${ }^{6}$ ICREA, Universitat Pompeu Fabra, Spain.
}

\begin{abstract}
Pre-adolescents who had suffered from intrauterine growth restriction (IUGR) during their mothers' pregnancy usually present more spherical hearts (smaller relation between base to apex measure and basal diameter), measured using echocardiograms, which has been associated with long-term cardiac disfunction. The present work aims to analyse these heart morphology changes by means of the surface ECG so as to have an early diagnostic tool of this pathology. The dataset is conformed by 148 preadolescents with either preterm or term births, and with or without IUGR. Once QRS and T-wave loops were obtained from the vectorcardiogram, the angles between the dominant vector of the $Q R S$ loop and $-X Y$ or $-Y Z$ planes $\left(\Phi_{\mathrm{R}-\mathrm{XY}}\right.$, $\left.\Phi_{\mathrm{R}-\mathrm{YZ}}\right)$ and the difference between $\Phi_{\mathrm{R}-\mathrm{XY}}$ and the angle between the dominant vector of T-wave loop ( $\left.\Phi_{\mathrm{T}-\mathrm{XY}}\right)$ and the $X Y$-plane showed different values for pre-adolescents who suffered from premature birth and IUGR than for control subjects $(p<0.05)$. These characteristics can open the door for a much easier diagnosis and follow-up of candidates for these disfunctions.
\end{abstract}

\section{Introduction}

Preterm birth refers to those fetuses who are born before 37 completed weeks of pregnancy. Besides preterm birth, intrauterine growth restriction (IUGR) is one of the main causes of low birth weight [1]. IUGR is diagnosed when a fetus presents poor growth in their mother's womb during pregnancy (i.e. he does not develop the whole growth potential). It is characterized by weight and mass lower than normal with respect to the number of gestational weeks below the tenth percentile $[2,3]$. Due to its high prevalence, it is a major cause of perinatal morbidity and mortality [4] and complicates about $8 \%$ of pregnancies.

Numerous studies have shown a clear correlation between low birth weight and presence of cardiovascular diseases in adulthood [5,6], even including cardiovascular mortality [7]. As cardiovascular diseases usually undergo a long subclinical period before the first clinical symptoms, it is of paramount importance to detect their risk as early as possible.

Recent studies have shown the effects of IUGR in the long-term (along postnatal life), which include heart remodeling and vascular disfunction and increased blood pressure [8]. In particular, more globular hearts, reduced longitudinal motion and impaired relaxation were observed on cohorts of fetuses and children who suffered from IUGR in their mothers' womb $[9,10]$.

In this paper we have studied if these characteristics can be observed in the surface electrocardiogram. Our objective is to identify features that could report differences in premature birth or IUGR along adolescence, which may be due to their more spherical hearts.

\section{Materials and Methods}

\subsection{Materials}

The study population is conformed by 148 preadolescents, whose surface 12-lead ECG was recorded at a sampling rate of $1000 \mathrm{~Hz}$ in a tertiary centre. 56 preadolescents suffered from IUGR inside the womb whereas 
92 pre-adolescents did not. On the other hand, 65 of the 148 pre-adolescents had a premature birth whereas the rest were born after a full-term pregnancy. Control group has been defined as subjects who had term birth and were adequate for gestational age (AGA), i.e. did not present IUGR. Below Table 1 shows the distribution of the cohort of subjects for which the study has been conducted.

Table 1. Subgroups distribution of the 148 subjects included in the study.

\begin{tabular}{c||c|c||c|c}
\hline \hline \multicolumn{1}{c||}{} & \multicolumn{2}{c||}{ Term } & \multicolumn{2}{c}{ Preterm } \\
\cline { 2 - 5 } & Male & Female & Male & Female \\
\hline AGA & 32 & 28 & 13 & 19 \\
\hline IUGR & 11 & 12 & 12 & 21 \\
\hline \hline
\end{tabular}

\subsection{Feature extraction}

\section{Dominant vectors of QRS and T loops}

First, ECG is delineated using wavelet transform, so that each peak of the individual waves (QRS complex and T wave) as well as their onset and end are detected [11]. Then, once baseline wander is reduced on the original ECG signal, the vectorcardiogram of length $K$ samples is obtained by means of the inverse Dower matrix [12].

Next, both depolarization and repolarization loops are aligned with respect to the first available loops, respectively. For those alignments, the method presented in [13] is used, so that the influence of the respiratory cycles is also removed.

Thus, three different transformations are considered for the above-mentioned alignment: scaling (for loop expansion or contraction), rotation (due to rotational changes of the heart because of, for example, respiration), and shifts (to obtain time synchronization). The equation that summarizes this process can be described by

$$
\boldsymbol{Z}=\alpha \boldsymbol{Q} \boldsymbol{Z}_{\boldsymbol{R}} \boldsymbol{J}_{\boldsymbol{\tau}}
$$

where $Z$ and $Z_{R}$ refer to the $3 \times K$ matrices of the aligned and the reference loops, respectively; $\alpha$ is the positive-valued parameter that allows scaling; $Q$ is the $3 \times 3$ rotation matrix, and $\boldsymbol{J}_{\boldsymbol{\tau}}$ is the shift matrix defined by the integer time shift $\tau$. For a fully detailed explanation of the alignment process (see [13]).

Once alignment has been done, for each vectorcardiogram it is obtained an average loop for each depolarization and repolarization set of loops. Figure 1 depicts an example of the average depolarization and repolarization loops for a pre-adolescent who suffered from IUGR, whereas Figure 2 depicts same features for a pre-adolescent used as control (had term birth and was AGA).
Then, we finally estimate the dominant vectors for both the average depolarization and repolarization loops of each recording by averaging the set of vectors composing each loop, which describe the dominant direction of the electrical wavefront during the QRS complex and the T-wave.

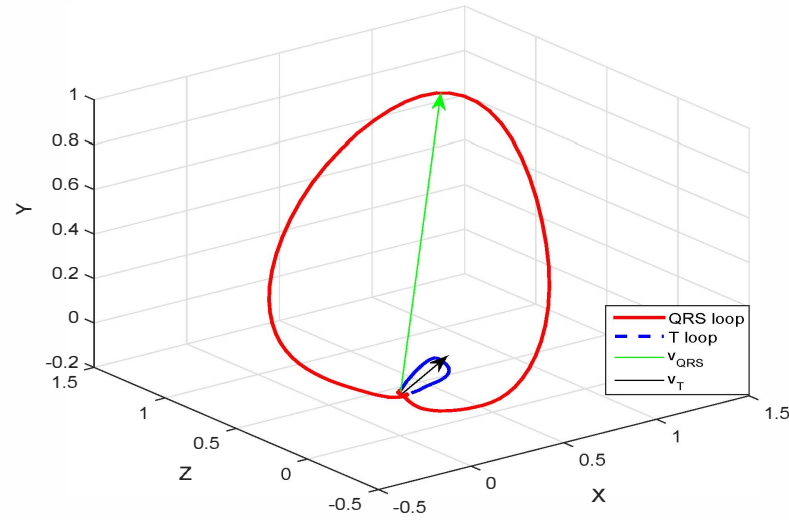

Figure 1. Average depolarization (QRS) and repolarization (T) loops for a female subject included in the study, who suffered from IUGR inside her mother's womb, and the respective dominant vectors.

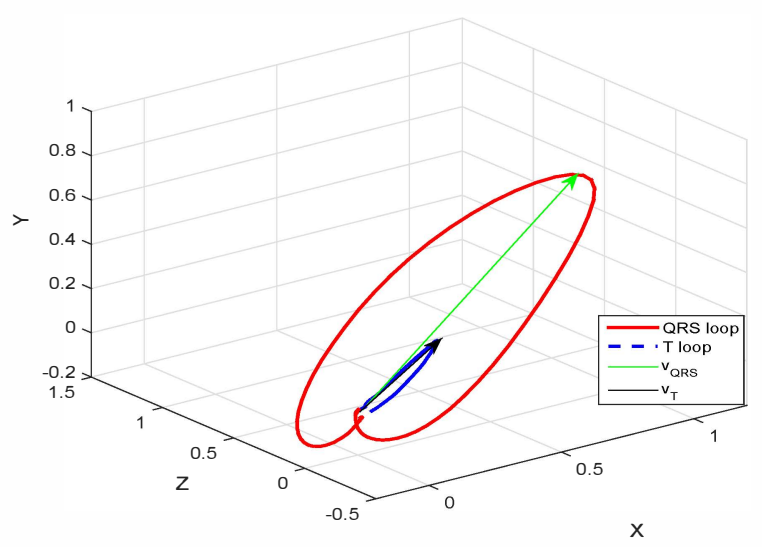

Figure 2. Average depolarization (QRS) and repolarization (T) loops and the respective dominant vectors for a female subject included in the study who had birth term and was AGA.

\section{Angles estimation}

Once we have obtained the dominant vectors for each average loop (denoted by $\boldsymbol{v}_{\mathrm{QRS}}$ and $\boldsymbol{v}_{\mathrm{T}}$ for the depolarization and repolarization loops, respectively) we proceeded to obtain some features in order to characterize and dif- 
ferentiate recordings corresponding to premature birth and IUGR pre-adolescents from control subjects.

First, we have estimated the angle between repolarization and depolarization dominant vectors in the threedimensional space $\left(\theta_{R T}\right)$. This is the angle between the QRS and T axis in the plane that they form, which commonly differs from the angle of the projections of QRS and $\mathrm{T}$ axes in the frontal plane.

In addition, we have also estimated the absolute angles between the above-mentioned dominant vectors $\boldsymbol{v}_{\text {QRS }}$ and $\boldsymbol{v}_{\mathrm{T}}$ and the three orthogonal planes $\mathcal{P} \in\{X Z, X Y, Y Z\}$, denoted by $\Phi_{\mathrm{R}-\mathcal{P}}, \Phi_{\mathrm{T}-\mathcal{P}}$, as well as the difference between these angles within the same plane.

Results obtained for each parameter are detailed in the following section.

Table 2. Results for the 148 subjects included in the study, differentiating among control subjects (termAGA), and subjects who belong to subgroups that present term/preterm and AGA/IUGR characteristics. Boldface indicates significant differences, please see text.

\begin{tabular}{|c|c|c|c|c|}
\hline Feature & $\begin{array}{l}\text { Term } \\
\text { AGA }\end{array}$ & $\begin{array}{c}\text { Term } \\
\text { IUGR }\end{array}$ & $\begin{array}{c}\text { Preterm } \\
\text { AGA }\end{array}$ & $\begin{array}{c}\text { Preterm } \\
\text { IUGR }\end{array}$ \\
\hline$\Phi_{\mathrm{R}-\mathrm{XZ}}$ & 35.38 & 35.21 & 32.00 & 34.21 \\
\hline Male & 34.32 & 36.97 & 31.24 & 34.50 \\
\hline Female & 36.59 & 33.60 & 32.52 & 34.05 \\
\hline$\Phi_{\mathrm{R}-\mathrm{XY}}$ & 19.17 & 22.55 & 24.63 & 25.55 \\
\hline Male & 19.12 & 19.81 & 28.61 & 29.82 \\
\hline Female & 19.23 & 25.06 & 21.91 & 23.11 \\
\hline$\Phi_{\mathrm{R}-\mathrm{YZ}}$ & 46.09 & 44.65 & 45.86 & 43.07 \\
\hline Male & 46.99 & 44.46 & 44.28 & 39.46 \\
\hline Female & 45.05 & 44.83 & 46.94 & 45.12 \\
\hline$\Phi_{\mathrm{T}-\mathrm{XZ}}$ & 35.13 & 33.41 & 33.78 & 33.88 \\
\hline Male & 35.79 & 36.47 & 32.11 & 36.10 \\
\hline Female & 34.38 & 30.61 & 34.76 & 32.60 \\
\hline$\Phi_{\mathrm{T}-\mathrm{XY}}$ & 19.54 & 21.89 & 22.01 & 18.78 \\
\hline Male & 22.14 & 22.84 & 25.25 & 19.75 \\
\hline Female & 16.58 & 21.02 & 19.79 & 18.22 \\
\hline$\Phi_{\mathrm{T}-\mathrm{YZ}}$ & 46.57 & 46.85 & 46.37 & 48.96 \\
\hline Male & 44.26 & 43.64 & 45.53 & 45.70 \\
\hline Female & 49.20 & 49.79 & 46.94 & 50.82 \\
\hline$\Phi_{\mathrm{R}-\mathrm{XZ}}-\Phi_{\mathrm{T}-\mathrm{XZ}}$ & 0.24 & -2.20 & -1.68 & 0.34 \\
\hline Male & -1.48 & 0.82 & -2.24 & -1.60 \\
\hline Female & 2.66 & -4.97 & -0.86 & 1.45 \\
\hline$\Phi_{\mathrm{R}-\mathrm{XY}}-\Phi_{\mathrm{T}-\mathrm{XY}}$ & -0.37 & 0.66 & 2.62 & 6.77 \\
\hline Male & -3.02 & -3.02 & 2.12 & 10.07 \\
\hline Female & 2.66 & 4.05 & 3.36 & 4.88 \\
\hline$\Phi_{\mathrm{R}-\mathrm{YZ}}-\Phi_{\mathrm{T}-\mathrm{YZ}}$ & -0.48 & -0.49 & -0.51 & -5.89 \\
\hline Male & 2.73 & 1.58 & -1.24 & -6.23 \\
\hline Female & -4.15 & -2.47 & -0.01 & -5.70 \\
\hline
\end{tabular}

\section{Results and Discussion}

In Table 2 we can observe results for the several angles measured using the dominant vectors of the depolarization and repolarization loops for each subgroup, considering as control group those subject who were born at term and were adequate for gestational age. In Table 3 we can observe the Student's t-test $\mathrm{p}$-values for each subgroup compared to the control group. Both tables remark in bold those results which are statistically significant. We have not included detailed results for feature $\theta_{R T}$ due to the fact that, for our particular study, this feature does not offer significant differences.

In Tables 2 and 3 we can observe that $\Phi_{\text {R-XY }}$ is statistically significant to differentiate those pre-adolescents who had premature birth from the control ones, whereas and $\Phi_{\mathrm{R}-\mathrm{XY}}-\Phi_{\mathrm{T}-\mathrm{XY}}$ is also significant to distinguish those subjects

Table 3. Student's t-test $p$-values for the different features for each subgroup under study in comparison with control subjects (i.e. term-AGA). Boldface indicates significant differences, please see text.

\begin{tabular}{|c|c|c|c|}
\hline Feature & $\begin{array}{l}\text { Term } \\
\text { IUGR }\end{array}$ & $\begin{array}{l}\text { Preterm } \\
\text { AGA }\end{array}$ & $\begin{array}{c}\text { Preterm } \\
\text { IUGR }\end{array}$ \\
\hline$\Phi_{\mathrm{R}-\mathrm{XZ}}$ & 0.937 & 0.060 & 0.529 \\
\hline Male & 0.371 & 0.272 & 0.951 \\
\hline Female & 0.306 & 0.089 & 0.308 \\
\hline$\Phi_{\mathrm{R}-\mathrm{XY}}$ & 0.233 & 0.035 & 0.013 \\
\hline Male & 0.869 & 0.011 & 0.009 \\
\hline Female & 0.131 & 0.467 & 0.248 \\
\hline$\Phi_{\mathrm{R}-\mathrm{YZ}}$ & 0.511 & 0.911 & 0.133 \\
\hline Male & 0.406 & 0.323 & 0.021 \\
\hline Female & 0.943 & 0.537 & 0.979 \\
\hline$\Phi_{\mathrm{T}-\mathrm{XZ}}$ & 0.383 & 0.421 & 0.447 \\
\hline Male & 0.821 & 0.258 & 0.921 \\
\hline Female & 0.138 & 0.842 & 0.308 \\
\hline$\Phi_{\mathrm{T}-\mathrm{XY}}$ & 0.379 & 0.290 & 0.731 \\
\hline Male & 0.854 & 0.361 & 0.525 \\
\hline Female & 0.227 & 0.304 & 0.535 \\
\hline$\Phi_{\text {T-YZ }}$ & 0.909 & 0.925 & 0.259 \\
\hline Male & 0.857 & 0.712 & 0.679 \\
\hline Female & 0.861 & 0.370 & 0.528 \\
\hline$\Phi_{\mathrm{R}-\mathrm{XZ}}-\Phi_{\mathrm{T}-\mathrm{XZ}}$ & 0.495 & 0.328 & 0.964 \\
\hline Male & 0.559 & 0.856 & 0.972 \\
\hline Female & 0.798 & 0.053 & 0.748 \\
\hline$\Phi_{\mathrm{R}-\mathrm{XY}}-\Phi_{\mathrm{T}-\mathrm{XY}}$ & 0.780 & 0.330 & 0.017 \\
\hline Male & 0.999 & 0.144 & 0.008 \\
\hline Female & 0.772 & 0.903 & 0.557 \\
\hline$\Phi_{\mathrm{R}-\mathrm{YZ}}-\Phi_{\mathrm{T}-\mathrm{YZ}}$ & 0.549 & 0.989 & 0.027 \\
\hline Male & 0.641 & 0.289 & 0.038 \\
\hline Female & 0.829 & 0.141 & 0.569 \\
\hline
\end{tabular}


who were preterm and had IUGR. In both cases, subjects show larger angle values when they had premature births. On the contrary, it is much more difficult to distinguish dissimilarities when analysing the case of term-IUGR preadolescents versus the control ones.

Regarding the results for term-IUGR subjects, it is more difficult to establish clear differences between preadolescents who suffered from IUGR inside the womb but the pregnancy was considered at term from control subjects.

It is of paramount importance to remark those results obtained on the male subgroups, since features behave better in this case, obtaining even smaller p-values. This should be studied in detail as future work, since previous references have pointed out that despite the similar increase in general death rate for men and women who suffered from IUGR, which was inversely related to their lower birth weight, only men developed cardiovascular diseases in adult life [14].

Indeed, it will be interesting to enlarge of the dataset included in the study and consider possible trends in the results.

\section{Conclusions}

In this paper we have presented a study that points to the measure of the absolute and relative angles formed by the depolarization and repolarization loops with the orthogonal axis as features to distinguish between control preadolescent subjects and those who had a premature birth or suffered from IUGR inside the womb, which could be reflected in electrical remodeling.

Presented results show statistically significant differences in the case of premature births, and point out for future work to the analysis of the influence of the onset of IUGR (differentiating between early -before the 34th week of pregnancy- or late onset -after the 34th week-) when characterising those subjects.

\section{Acknowledgements}

N. Ortigosa acknowledges the support from Generalitat Valenciana under grant PrometeoII/2013/013 and MINECO under grant MTM2013-43540-P.

This project has also been funded by TEC2013-42140$\mathrm{R}$ and TIN2014-53567-R from CICYT, Spain, by Grupo Consolidado BSICoS from DGA (Aragón) and European Social Fund, and the Erasmus + Programme of the European Union (Framework Agreement number: 2013-0040). This publication reflects the views only of the authors, and the Commission cannot be held responsible for any use which may be made of the information contained therein.

\section{References}

[1] Diderholm B. Perinatal energy metabolism with reference to iugr and sga: studies in pregnant women and newborn infants. Indian J Med Res 2009;130:612-617.

[2] Committee on Practice Bulletins-Gynecology, American College of Obstetricians and Gynecologists, Washington, USA. Intrauterine growth restriction. Clinical management guidelines for obstetrician-gynecologists. American College of Obstetricians and Gynecologists. Int J Gynaecol Obstet 2001;72(1):85-96.

[3] Figueras F, Gratacós E. Update on the diagnosis and classification of fetal growth restriction and proposal of a stage-based management protocol. Fetal Diagn Ther 2014; 36(2):86-98.

[4] Alberry M, Soothill P. Management of fetal growth restriction. Arch Dis Child Fetal Neonatal Ed 2007;92:62-67.

[5] Barker D, Osmond C, Golding J, Kuh D, Wadsworth M. Growth in utero, blood pressure in childhood and adult life, and mortality from cardiovascular disease. BMJ March 1989;298:564-567.

[6] Barker D. Fetal origins of coronary disease. BMJ 1995; 311:171-174.

[7] Ueda P, Cnattingius S, Stephansson O, Ingelsson E, Ludvigsson J, Bonamy A. Cerebrovascular and ischemic heart disease in young adults born preterm: a populationbased swedish cohort study. Eur J Epidemiol April 2014; 29(4):253-60.

[8] Demicheva E, Crispi F. Long-term follow-upv of intrauterine growth restriction: cardiovascular disorders. Fetal Diagn Ther 2014;36:143-153.

[9] Crispi F, Bijnens B, Figueras F, Bartrons J, Eixarch E, Noble FL, A AA, Gratacós E. Fetal growth restriction results in remodeled and less efficient hearts in children. Circulation June 2010;121(22):2427-36.

[10] Cruz-Lemini M, Crispi F, Valenzuela-Alcaraz B, Figueras F, Gómez O, Sitges M, Bijnens B, Gratacós E. A fetal cardiovascular score to predict infant hypertension and arterial remodeling in intrauterine growth restriction. Am J Obstet Gynecol June 2014;210(6):552.e1-552.e22.

[11] Martínez J, Almeida R, Olmos S, Rocha A, Laguna P. A Wavelet-Based ECG Delineator: Evaluation on Standard Databases. IEEE Trans Biomed Eng 2004;51(4):570-581.

[12] Edenbrandt L, Pahlm O. Vectorcardiogram synthetized from a 12-lead ECG: Superiority of the inverse Dower matrix. J Electrocardiol 1988;21:361-367.

[13] Sörnmo L. Vectorcardiographic loop alignment and morphologic beat-to-beat. IEEE Trans Biomed Eng 1998; 45:1401-1413.

[14] Osmond C, Barker D, Winter P, Fall C, Simmonds S. Early growth and death from cardiovascular diseaase in women. BMJ 1993;307:1519-1524.

Address for correspondence:

Nuria Ortigosa

IUMPA, Universitat Politècnica de València

Camino de Vera s/n, 46022 Valencia (Spain)

nuorar@upvnet.upv.es 